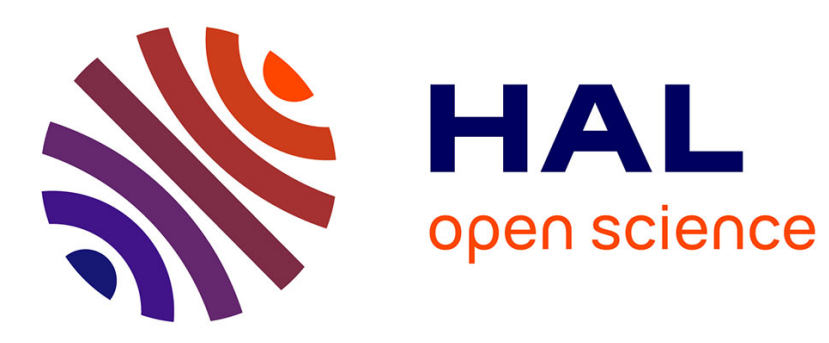

\title{
Fast Monte Carlo-based Inverse Planning for Prostate Brachytherapy by Using Deep Learning
}

Mateo Villa, Julien Bert, Antoine Valeri, Ulrike Schick, Dimitris Visvikis

\section{To cite this version:}

Mateo Villa, Julien Bert, Antoine Valeri, Ulrike Schick, Dimitris Visvikis. Fast Monte Carlo-based Inverse Planning for Prostate Brachytherapy by Using Deep Learning. IEEE Transactions on Radiation and Plasma Medical Sciences, 2021, pp.1-1. 10.1109/TRPMS.2021.3060191 . hal-03180735

\section{HAL Id: hal-03180735 \\ https://hal.science/hal-03180735}

Submitted on 25 Mar 2021

HAL is a multi-disciplinary open access archive for the deposit and dissemination of scientific research documents, whether they are published or not. The documents may come from teaching and research institutions in France or abroad, or from public or private research centers.
L'archive ouverte pluridisciplinaire HAL, est destinée au dépôt et à la diffusion de documents scientifiques de niveau recherche, publiés ou non, émanant des établissements d'enseignement et de recherche français ou étrangers, des laboratoires publics ou privés. 


\title{
Fast Monte Carlo-based Inverse Planning for Prostate Brachytherapy by Using Deep Learning.
}

\author{
Mateo Villa, Julien Bert, Antoine Valeri, Ulrike Schick and Dimitris Visvikis.
}

\begin{abstract}
Inverse planning is an essential tool for optimizing the delivered radiation dose on low-dose-rate (LDR) prostate brachytherapy. Clinical inverse planning systems use the TG-43 dose computation formalism in order to perform a fast optimization. However, this method is an approximation that often leads to a dose overestimation, resulting on sub-optimal plans. Alternatively, Monte Carlo simulation (MCS) can be used to obtain an accurate dose distribution, but considerably increasing the estimation time. We propose a fast inverse planning method for LDR prostate brachytherapy that uses a deep convolutional neural network (DCNN) trained on a GPU-based MCS generated database to estimate the dose distribution on the prostate and organs at risk. Segmentations of the organs and seeds' positions are given as the DCNN input. The mean percent error on the test set was $-1.19 \pm 0.94 \%$ within the prostate. The DCNN was used to estimate the dosimetric parameters in each organ for every configuration of the optimization loop. The dosimetric parameters of the final DCNN-based brachytherapy plans were in good agreement compared to the same plans recalculated with a full MCS. The proposed inverse planning based on DCNN was capable to reach an equivalent level of accuracy with Monte Carlo with a runtime in less than 1 min using conventional GPU card.
\end{abstract}

Index Terms-Brachytherapy, Dosimetry, Deep Learning, Inverse Planning

\section{INTRODUCTION}

B RACHYTHERAPY is an internal radiotherapy technique widely used on the treatment of the prostate cancer. It involves the application of radioactive sources through the prostate on the tumor proximity, with the aim of destroying the tumor while preserving healthy distant organs and tissues from the radiation exposure. Low-dose-rate (LDR) brachytherapy is one of the most popular for the case of low-stage prostate cancer. In this method, multiple small seeds of radioactive material such as iodine $125\left({ }^{125} \mathrm{I}\right)$ or paladium $103\left({ }^{103} \mathrm{Pd}\right)$ are implanted and remain permanently into the affected organ region. The seeds implantation is done by using hollow needles which are inserted through the perineum, with the guide of trans-rectal ultrasound (TRUS) imaging. The intervention is thus less invasive than in other kind of procedures such as external radiotherapy or high-dose-rate (HDR) brachytherapy.

Nevertheless, the spatial distribution of the inserted seeds during LDR brachytherapy is highly determinant on the resulting radiation dose. Therefore, an improper configuration plan may result on harmful side effects caused by high and

All the authors are with LaTIM, INSERM, Faculté de Médicine, IBRBS 22, Av. Camille Desmoulins, 29200 Brest, France. (email: ingmatvillaa@gmail.com)

This work was funded by the French National Research Agency through the FOCUS project (ANR-16-CE19-0011), the OptimiX project (ANR-18-CE450011), and within the Investissements d'Avenir program (LabEx CAMI 2) under reference ANR-11-LABX-0004. heterogeneous zones of radiation. For avoiding such issues, inverse planning is an essential tool for guiding LDR prostate brachytherapy routine. In this technique, an optimization algorithm searches the most suitable seeds locations by minimizing a cost function, which is composed by pre-defined dose objectives. The final evaluation of the procedure is made by using dose-volume histograms (DVHs), which give information about the dose homogeneity and intensity on the prostate and organs at risk (OARs), specifically the urethra and rectum. The DVHs must fill different criteria specified by the American Association of Physicist in Medicine (AAPM) on their Task Group (TG) TG-137 report [1]. Fast simulated Annealing (FSA) [2] is one of the most popular optimization algorithms used in practice. Other kind of approaches such as genetic algorithms [3], [4] and compressed sensing inspired solvers [5] have also been proposed. All of these methods allow to achieve plans in less than one minute. However, in the current clinical applications, the dose objectives are not optimized on the entire organs' volume, but only on points of the surface. As a result, clinical planning systems tend to obtain plans that does not fulfil the DVHs criteria entirely, and a manually correction by an expert radiologist should often be made, consequently increasing the time and risk of the intervention. DVH-based optimization has been successfully implemented on inverse planning systems for external radiotherapy, but they are not yet used in real practice on prostate LDR brachytherapy due to its elevate time computation.

Moreover, clinical inverse planning systems compute the dose distribution based on the TG-43 formalism, an analytical model established by the AAPM [6], [7]. This method, regardless of allowing a fast computation, it does not consider the organs densities and the interaction between the sources, causing an overestimation of the dose [8]-[10], that could reach up to $12 \%$ for the $D_{90}$ parameter (the dose absorbed by at least the $90 \%$ of the prostate volume) [9]. Monte Carlo (MC) dosimetry can be used to obtain a more precise estimation. Nevertheless, computation time is not suitable for a clinical application. Different Monte Carlo simulation (MCS) frameworks based on Graphics Processing Units (GPU) have been proposed [11][14], and they proved a reduction on computation time from multiple hours to seconds for a single dose map. Even though, the inverse planning algorithms usually perform a thousand of iterations before obtaining a satisfactory solution, leading to a very long-time calculation to get the final optimized plan. A DVH-based optimization algorithm using MCS dosimetry for LDR prostate brachytherapy has previously proposed [15]. In this work, MCS is used to compute dose kernels for each single seed position, followed by the use of FSA. At each optimization step, the current dose kernel is obtained by 
adding the dose distribution of the selected seeds. This method achieved treatment plans within less than one minute, with most of the DVH critical values matching the criteria. Still, this method does not include the effect of seeds' interaction, and the need of storing a large data set of dose kernels may rise issues on memory management and input/output time access. Additionally, the number of simulated particles used to estimate each dose kernel had to be reduced in order to achieve a fast planning, which was reflected in an increase of uncertainty.

On the other hand, deep convolutional neural networks have been broadly used in the last years in many medical imaging applications due to their ability to accurately learn a mapping between an input image and corresponding output. Recent works in the field of radiotherapy and medical imaging dosimetry have been released, with the aim to predict the resulting dose in to the patient's body. The general approach consist to train a deep convolutional neural network (DCNN) with a medical image (CT, PET, MRI) or a segmentation map of the organs as the input and the desired dose map as an output. In a recent work [16], a DCNN was trained to learn the dosimetry prediction in HDR brachytherapy for a given treatment plan, based on the patient's image and organs' segmentation. However, due to time computation to build a large training data set from MCS, they pre-train a network using water-based dose. The dose prediction time was 1.7 seconds. In HDR there is only one source at the time within the patient and the source only interacts with human tissues. Such method cannot be used in LDR, since the interactions between the seeds have to be considered. The water-based pre-training method may introduce bias because the dose in water and seed are very different. In addition, the optimization in LDR needs a large number of iterations to explore every possible seed configuration. A much faster dose prediction is required in LDR.

We propose in this paper a fast inverse planning method for LDR prostate brachytherapy that uses a deep convolutional neural network (DCNN) trained on a GPU-based MCS generated database to estimate the dose distribution on the prostate and organs at risk (OARs). Segmentation maps of the concerned organs and seeds' positions are given as the input of the DCNN, and the dose distribution obtained by Monte Carlo simulation is the output to be learned. The DCNN was used to estimate the dosimetric parameters in each organ for every configurations test by the optimization loop.

\section{Methods And MATERIAls}

We propose the use of a DCNN which learns to compute the dose into the prostate and OARs for a given patient anatomy and set of seeds' positions. Figure 1 show a slice example of the input and output. A fast GPU-based MCS is used to generate the dose maps database. The trained model is then used to obtain the dose at each step of the inverse planning. The following sections explains the approach in detail.

\section{A. Patient's Database}

Prostate and OARs contours from 273 ultrasound (US) image patients who were undergone with LDR brachytherapy
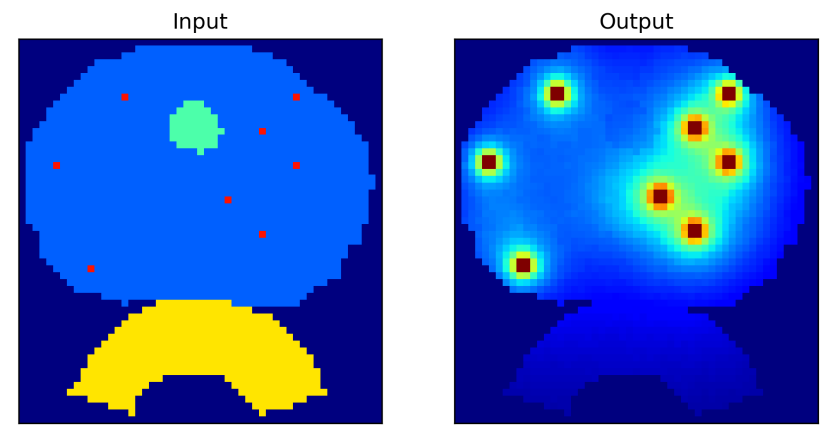

Fig. 1. Example slice of input (left) and output (right) of the DCNN-based method. The light blue label represents the prostate, the light green the urethra, the yellow the rectum, and the red points the seeds of the current configuration. The output used for training is the dose map obtained from MCS.

at University of Brest Hospital were extracted. The whole data set contains prostate volumes with sizes varying between 17 $\mathrm{cm}^{3}$ and $67 \mathrm{~cm}^{3}$.

The original ultrasound data had a size of $550 \times 550 \times 88$ voxels with a pixel spacing of $0.15 \mathrm{~mm} \times 0.15 \mathrm{~mm}$ and a slice thickness of $1 \mathrm{~mm}$.

For each US image patient, 50 layout of seed positions were generated randomly, with a number of seeds varying randomly between 48 and 70. This lower and upper values were obtained according the number of seeds usually implanted to the patient, which depends on the prostate volume size. The space of possible positions follows the standard grid of $5 \mathrm{~mm}$ spacing used during the needles' insertion. The resulting number of configurations was 13650 for the entire data set.

\section{B. MC Dose Computation}

Since MCS usually involves long time computation, a generation of a large dose map data set would be difficult with conventional MCS software. Therefore, dose distributions on the prostate and OARs were generated by using GGEMS, a validated GPU-based MC simulation platform for dosimetry applications including LDR brachytherapy [13], [14]. The simulation was performed by using ${ }^{125}$ I sources at the desired positions on a voxelized phantom generated from the patient contours. The complete geometry of the seeds (STM1251, Bard Medical Division, Covington, GA, USA) was taken into account, similarly to [14]. For each organ label (prostate, urethra, rectum) the corresponding material composition was assigned, based on the recommendations of the International Commission on Radiation Units and Measurements Report No. 46 [17]. The surrounding area of the aforementioned organs is considered as a soft-tissue and its material is also included in order to fully take into account tissue heterogeneities. GGEMS software was based on Geant4 physics effects. The GPUbased MC simulation was setup to use only photon physics effects without considering electrons. Electron ranges for the ${ }^{125}$ I sources are very small compared to the size of a voxel [18]. Therefore, their doses were deposited locally within each voxel they were emitted from. The dose calculation was performed using the track-length estimator [19] which is a variance reduction technique allowing to reduce time 
computation without introducing any approximations. A total of $5 \times 10^{7}$ particles were simulated, allowing to achieve approximately $0.5 \%$ mean statistical uncertainty per dose map on the prostate volume, $0.6 \%$ on the urethra and 1 $\%$ on the rectum. Simulations took on average 20 seconds approximately per configuration on a NVIDIA GTX 1080 GPU.

\section{Deep Convolutional Neural Network (DCNN)}

We propose a DCNN model that uses as input the patient's 3D segmentation map, composed by the prostate, urethra, and the rectum. Additionally, the different seeds positions were directly encoded on the image by defining a 1-pixel label for each seed and included on the organs' segmentation map, as shown in the figure 1 . In order that the network considers organ structures and seed positions, all information was numerically encoded by using a label value inside the input image. For example, all voxels containing prostate tissue provided by the segmentation were set to the label value 1 . The same for the urethra and the rectum with label value 2 and 3 respectively. Voxels containing soft tissue were filled with the value zero. The voxel that corresponds to a seed position was filled with a label value 4. An example of final labelled image is illustrated on figure 1 (see the input image). Contour of each organ was achieved by the urologist using the transrectal US image while the patient is under general anesthesia in operating room. Then an inverse planning is run to determine an optimal plan, this is the standard clinical workflow on prostate brachytherapy. The seeds label will be changed during the optimization process, when a new seed position is explored, the current seed position is cleared by filling the corresponding voxel this prostate label, and the voxel that will receive the new seed position is filled with the label 4.. The output of the network will be the prediction of 3D dose distribution according organ contours and a given seed layouts.

1) DCNN Architecture: We designed a DCNN based on the 3D-Unet [20], which was originally intended for volumetric semantic segmentation. The architecture, which is shown in Figure 2 consist of two main stages: the contracting path (encoder), which extracts different features of the images by applying 3D convolutional filters and reduces their dimensionality using 3D max-pooling layers; and the expanding path (decoder), which gradually up-samples the images by using 3D deconvolutions (transposed convolutions) while concatenating them with the different features of the contracting path, until arriving to an output of the same dimension of the input. The main difference with the original architecture lies in the number of filters per convolution layer, which in our case were reduced by a factor of 8 , in order to avoid over-fitting and being able to have a fast prediction. Additionally, the use of a Rectified-Linear-Unit (ReLU) activation layer instead of the softmax layer (which is more suitable for classification) at output layer enabled the network to be trained for this regression task.

The original images were re-sampled to a resolution of 1 $\mathrm{mm} \times 1 \mathrm{~mm} \times 1 \mathrm{~mm}$ (the slice thickness did not change) in order to allow the network to perform a fast inference, leading to image with input dimensions of $88 \times 88 \times 88$ voxels. These images were centered at the prostate isocenter and a zeropadding was performed in order to match the input size of the neural network.

2) Training and Validation: The data set was divided patient-wise in a stratified fashion according to 6 ranges of prostate sizes. Training, validation, and test sets were created by randomly taking $60 \%, 20 \%$ and $20 \%$ of the patients of each interval, as shown in Figure 3. This strategy allows to have a representative sample of the data set on each of the splits, thus reducing the risk of over-fitting. The data split results on 163, 55 and 55 patients for each set. With the 50 generated plans per patient (and corresponding dose maps), the final split consists of 8150 data for train, 2750 for validation and 2750 for test.

The training phase was made using adaptive momentum algorithm (ADAM) [21] for minimizing the mean squared error (MSE) between the predicted dose and the MCS dose. During the network training, both training and validation loss were monitored. Consequently, the train process was stopped at the epoch when the validation loss ceased showing improvement with respect to training loss (i.e., the $17^{\text {th }}$ epoch), as shown in figure 4. The architecture was developed and trained on Keras, using Tensorflow back-end, using a Nvidia GeForce RTX 2080Ti GPU.

\section{DVH-based FSA for Inverse Planning}

Inspired by the work of [15], we performed an inverse planning based on dose-volume-histograms (DVHs) by using the Fast Simulated Annealing (FSA) algorithm [2]. The cost function to minimize, given by the equation 1 , includes the different DVH dosimetric criteria given by the AAPM TG137 recommendations [1]:

$$
\begin{aligned}
C= & w_{v 100} * \Theta\left(V_{100_{L}}-V_{100}\right)+ \\
& \sum_{i} w_{v i} \Theta\left(V_{i}-V_{i_{H}}\right)+\sum_{j} w_{d j} \Theta\left(D_{j}-D_{j_{H}}\right)+ \\
& \sum_{k} w_{d k} \Theta\left(D_{k}-D_{k_{H}}\right)+w_{N_{\text {needles }}} * N_{\text {needles }}
\end{aligned}
$$

where $V_{i}$ represents the percent of the prostate volume that receives at $i \%$ of the prescribed dose, $D_{j}$ represents the percent of the prescribed dose absorbed by $\mathrm{j} \%$ of the urethra's volume, and $D_{k}$ the amount of dose received in $\mathrm{kcm}^{3}$ of the rectum's volume. The subscripts $H$ and $L$ represent the critical values ( $\mathrm{L}$ for lowest, $\mathrm{H}$ for highest) of the aforementioned metrics. $\mathrm{N}$ is the number of needles used to insert the seeds. In the same way $w_{v i}, w_{d j}, w_{d k}$ and $w_{N}$ are weight factors given to each metric. Here, in order to give the same importance to all parameters, each weight was equally distributed i.e. with a value of $1 / N_{w}$, where $N_{w}$ is the number of weights uses in the cost function. $\Theta(X)$ represents the heavyside step function that overrides the cost contribution when its value is negative. The indices values are $i=[150,200]$ and $j=[10,30]$ and $k=[2 c c, 0.1 c c]$.

Before optimization, a fixed number of seeds is selected for the current patient, using the equation introduced by [22]: 


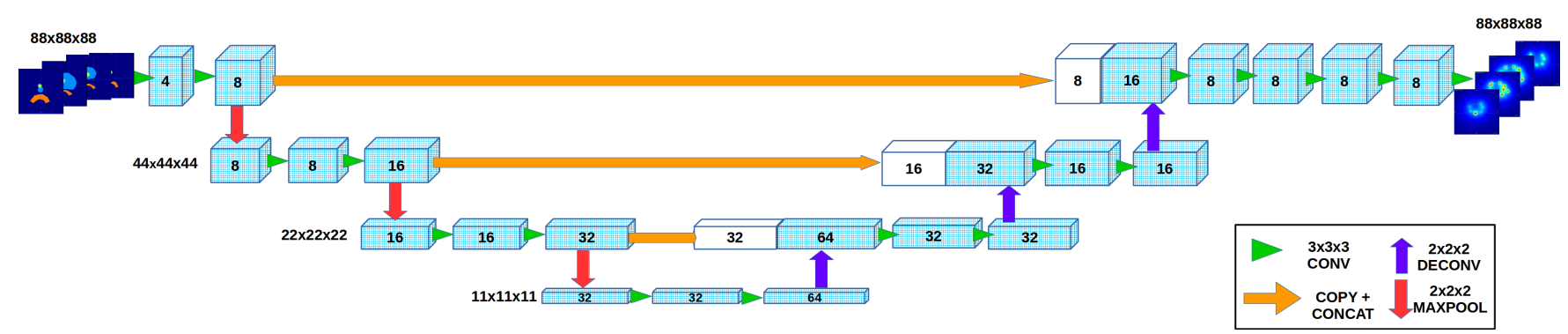

Fig. 2. Architecture of the proposed DCNN model.

Prostate volume distribution along train,validation and test sets

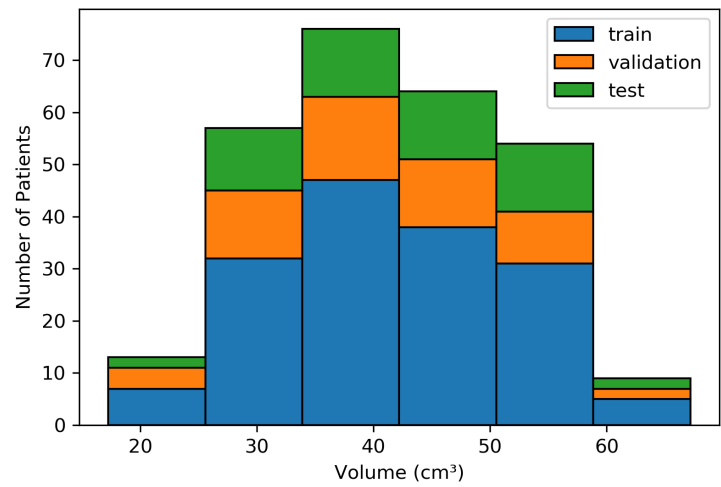

Fig. 3. Patients' prostate volume distribution along the training, validation and test sets

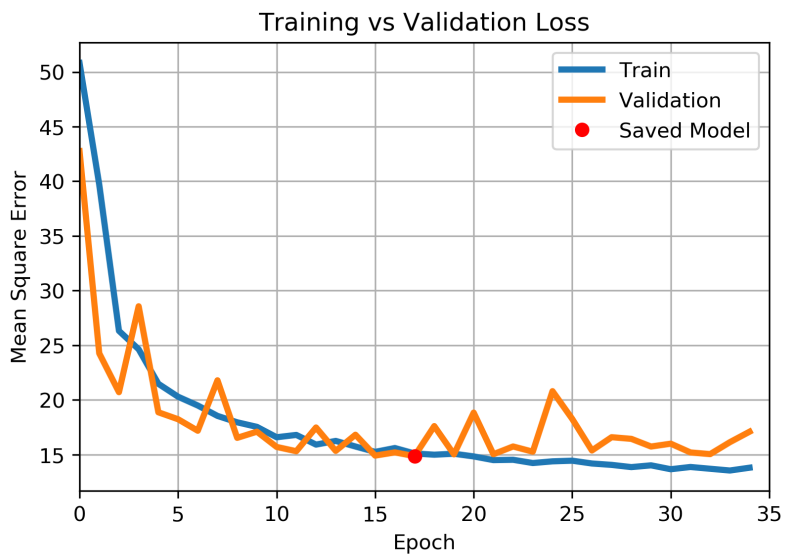

Fig. 4. Plot of train vs. validation loss as a function of epochs. The red dot indicates the epoch with the lowest error, which model weights were kept for the test set evaluation.

$$
N_{\text {seeds }}=4+\frac{4.674 \times V^{0.562}}{A}
$$

where $\mathrm{V}$ is the volume of the prostate, and $\mathrm{A}$ represents the strength of the source. The FSA outline starts with a random configuration of seeds. The resulting dose map is estimated using our trained DCNN and then the corresponding DVHs from the prostate, urethra and rectum are extracted. The cost function is calculated with the derived DVH dosimetric values. For the following steps, each configuration is obtained by randomly changing a single seed position. If the cost of the new configuration is lower than the previous one, then the new one is maintained. Differently, the new configuration is accepted with the following probability:

$$
P(\Delta C)=\exp (-\Delta C / T(k))
$$

Where $\mathrm{k}$ is the current optimization iteration, $\Delta C$ is the difference between the current and previous cost function, and $\mathrm{T}$ is the annealing temperature at the $k^{\text {th }}$ iteration. The temperature is changed at each step by applying a pre-defined cooling-rate factor between 0 and 1. The optimization takes end after a pre-defined number of iterations. This schedule makes that at the first iterations, non-conform configurations could be chosen, allowing to explore a wide range of solutions and avoiding the algorithm to get stuck on a local minima. In our study, we empirically set $\mathrm{T}=10^{5}$ and $\mathrm{C}=1 \%$ and $\max$ 2000 iterations. We set a fixed source strength of $0.645 \mathrm{U}$ for all the optimizations, which corresponds to the average of the sources used in the real plans. The DVHs were calculated with the same prescribed dose of the real treatments, which is equal to $160 \mathrm{~Gy}$.

\section{E. DCNN Dose Prediction Evaluation}

The trained model was used to estimate dose maps on the 2750 images of the test set, which for recall, correspond to 50 random configurations of 55 patients. The quality of the predicted dose maps was assessed in terms of the mean percent error (MPE) between the prediction and the ground-truth for each organ, which is defined as follows:

$$
M P E_{\text {organ }}=\frac{100 \%}{N_{\text {organ }}} \sum_{i} \frac{D_{i}^{\text {pred }}-D_{i}^{M C}}{D_{i}^{M C}}
$$

Where $D_{i}^{\text {pred }}$ and $D_{i}^{M C}$ represents the absorbed dose on the $i$ voxel of the organ for the prediction and the MC dose map, and $N_{\text {organ }}$ is the total number of voxels of the organ. We report the mean and the standard deviation of the MPE across the entire test set for each organ, as well as the graphical distribution in terms of the median, first and third quartiles, and first and ninth deciles.

\section{F. Inverse Planning Quality Evaluation}

The quality of the inverse planning has been evaluated on the 55 patients of the test set. For each patient, using the 
segmented organs, an inverse planning was performed using the FSA algorithm with our trained DCNN model as the dose calculation model. For each final optimized plan, the seed configuration was exported and used to recalculate a dose map using Monte Carlo simulation with the same parameters presented in II-B. DVH parameters were estimated on both dose map, the one directly obtained by the DCNN-based inverse planing after optimization and on the one recalculated by Monte Carlo simulation. The aim is to assess if after a DCNNbased optimization the equivalent MCS DVH parameters in terms of the TG-137 criteria are fulfilled. The DVH parameters are always calculated with respect to a prescribed dose of 160 Gy, which is the standard dose applied on the real treatments of the database.

\section{RESULTS}

\section{A. DCNN Evaluation}

Training of the DCNN was run on approximately 12 hours and dose prediction was achieved in approximately $38 \mathrm{~ms}$ per dose map on a Geforce RTX 2080 Ti GPU, which is highly faster than the 20 seconds of the MCS.

The distributions of the MPE of the DCNN dose prediction for the prostate and OARs along the test set are shown on figure 6. The mean \pm STD MPE for the entire test set are $-1.19 \% \pm 0.94 \%, 0.52 \% \pm 1.42 \%$, and $-1.47 \% \pm 1.90 \%$ on the prostate, urethra and rectum respectively. According to this results, the DCNN shows a general tendency to underestimate the dose in the prostate and the rectum, while in the urethra the dose is generally over-estimated. Nevertheless, the mean error magnitude does not exceed the limit of $2 \%$ for the prostate and urethra, and $4 \%$ for the rectum.

In general it was found that the dose predictions were visually in good agreement with the ground-truth. The figure 5 shows a representative middle-slice example of dose distribution of one of the test set configurations, in comparison to the MCS dose map. We can observe again the general tendency to over-estimation of dose in the urethra, which appears to be predicted with similar values of the prostate neighboring region. In the case of the prostate, we confirm the general trend of under-estimation of the dose, especially in zones of low and middle dose. For the rectum, most of the doses are under-estimated. Despite the fact that some pixels of the image may have errors reaching the $8 \%$, the absolute difference is still relatively low, and the accuracy the overall image and different organs is not significantly affected.

\section{B. Inverse Planning Quality}

The FSA algorithm achieved acceptable configurations in 2000 iterations on average. Adding to the DCNN prediction time (38 ms per dose map) to the time taken by the DVHs extraction and cost function calculation, the total optimization time was on average 1 minute. Which was very fast considering that dose maps accuracy were close to a fully Monte Carlo simulation.

The table I summarizes the different DVH parameters obtained with our DCNN inverse planning approach and their equivalent by running MCS on the resulting plans, as well as the TG-137 criteria for each parameter, with respect to reference prescribed dose of $160 \mathrm{~Gy}$. We can observe an underestimation of the dose for the $V_{100}$ and the $V_{150}$ which does not exceed the $1 \%$ and a very reduced over-estimation of the $V_{200}$ of $0.2 \%$. For the urethra, the $D_{10}$ and $D_{30}$ does not exceed $2 \%$ of difference with the resultant MCS. For the case of the rectum, we can observe an under estimation of the $D_{0.1 c c}$ of $3 \%$, whereas the $D_{2 c c}$ is predicted above $0.4 \%$ of the MCS value, which is still non-significant. In spite of those differences, it can be seen that all the DVH parameters recalculated by MCS on the final configuration complied the TG-137 criteria.

The figure 7 shows a representative example of the DVHs of an optimized plan by using the DCNN dose calculation, compared with the DVHs obtained after doing MCS on the final optimized configuration. The DCNN DVHs fit almost the entire MCS DVHs despite of having the over and underestimation behaviors highlighted previously. Finally, figure 8 shows a comparison of the iso-doses of (contours of the dose map containing the same value of dose) of both DCNN and the resultant MCS, for another typical case. The levels of the iso-doses were chosen based on the usual values analyzed by the radiologists. The DCNN iso-doses match accurately with the MCS, which confirms again that the DCNN has the capability to match the MCS dose distribution in terms of dose homogeneity.

\section{DISCUSSION}

The generalization capability of a DCNN model depends primarily on the network model parameters, training hyperparameters, the quality of the data set, and the way it is split for training, validation and test. Therefore, we set the number of convolutional filters per layer as small as possible without losing accuracy with the aim to prevent over-fitting and perform fast predictions. On the other hand, we needed to split the patients in a way that the three ensembles had a good representation of the entire data set. Despite the fact that the geometry of the prostate is relatively consistent, we found that the volume size was substantially variable from one patient to another, and we thus decided to use it as the criteria for splitting. This stratified approach contributed significantly to avoid to over-fitting, and moreover made easy the hyperparameter tuning.

According to the dose prediction results, both errors on the prostate and the urethra were below $2 \%$, and rectum error was below $5 \%$, which is a good indicator of the performance of our method. The lower accuracy on the rectum's dose prediction may be caused by different reasons. First of all, the rectum is generally more distant to the sources than the prostate (which contains all of them) and the urethra (which is located within the prostate region), which is reflected in less radiation dose. Since the DCNN optimizer tends to minimize the mean square error, then differences in low doses would be less relevant on the weights updating than the high doses errors. Another phenomenon frequently observed is that the DCNN has a smoothing effect in regions where the dose level changes in a considerable proportion, as observed in 

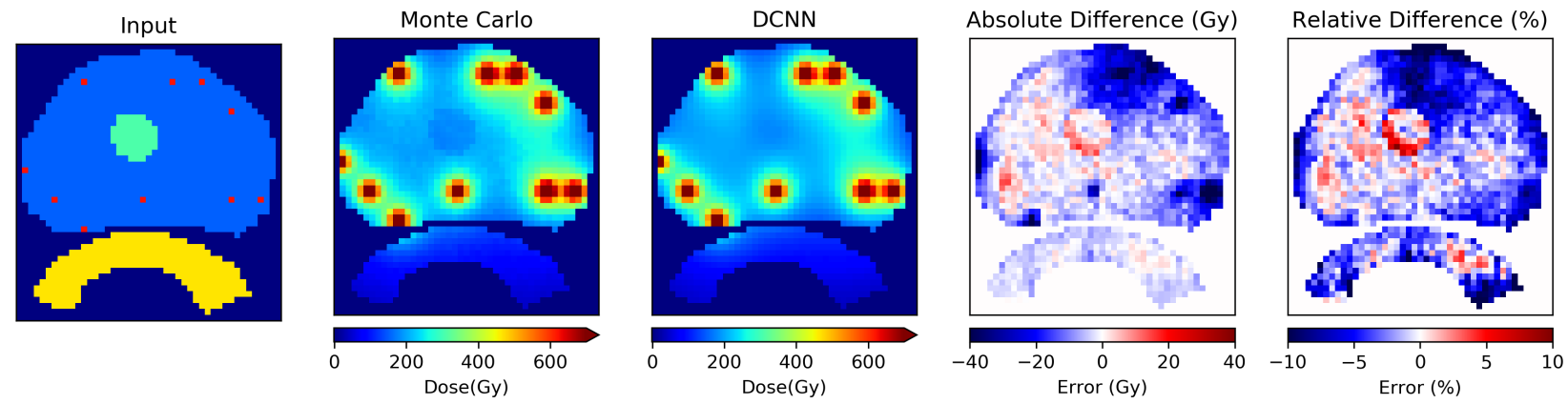

Fig. 5. Dose distribution comparison on an intermediate slice for a test set patient. From left to right: The input of the network (segmented organs and seeds), the ground-truth dose map obtained through MCS, the dose map predicted by our DCNN model, the absolute and the relative difference between the predicted and the groun-truth dose.

TABLE I

EVALUATION OF THE DVH PARAMETERS RESULTING FROM INVERSE PLANNING USING THE DCNN DOSE CALCULATION METHOD WITH A REFERENCE PRESCRIBED DOSE OF $160 \mathrm{GY}$.

\begin{tabular}{|llcrrr|}
\hline Organ & DVH Metric & Criteria & \multicolumn{1}{c|}{ DCNN } & MCS (Ground-Truth) & \multicolumn{1}{c|}{ Dif (\%) } \\
\hline \multirow{3}{*}{ Prostate } & $V_{100}(\%)$ & $\geq 95$ & $95.1 \pm 0.3$ & $95.8 \pm 1.0$ & $-0.8 \pm 1.0$ \\
& $V_{150}(\%)$ & $\leq 50$ & $47.4 \pm 2.2$ & $48.1 \pm 4.4$ & $-0.6 \pm 3.1$ \\
& $V_{200}(\%)$ & $\leq 20$ & $19.9 \pm 1.5$ & $19.7 \pm 2.4$ & $0.2 \pm 1.3$ \\
\hline \multirow{2}{*}{ Urethra } & $D_{10}(\%)$ & $<150$ & $135.2 \pm 7.8$ & $133.5 \pm 8.0$ & $1.7 \pm 3.5$ \\
& $D_{30}(\%)$ & $<130$ & $123.2 \pm 5.3$ & $122.3 \pm 5.5$ & $0.9 \pm 2.7$ \\
\hline \multirow{2}{*}{ Rectum } & $D_{2 c c}(\%)$ & $<100$ & $71.2 \pm 7.8$ & $70.8 \pm 7.6$ & $0.4 \pm 2.6$ \\
& $D_{0.1 c c}(\%)$ & $<150$ & $112.1 \pm 15.8$ & $114.9 \pm 21.1$ & $-2.8 \pm 9.2$ \\
\hline
\end{tabular}

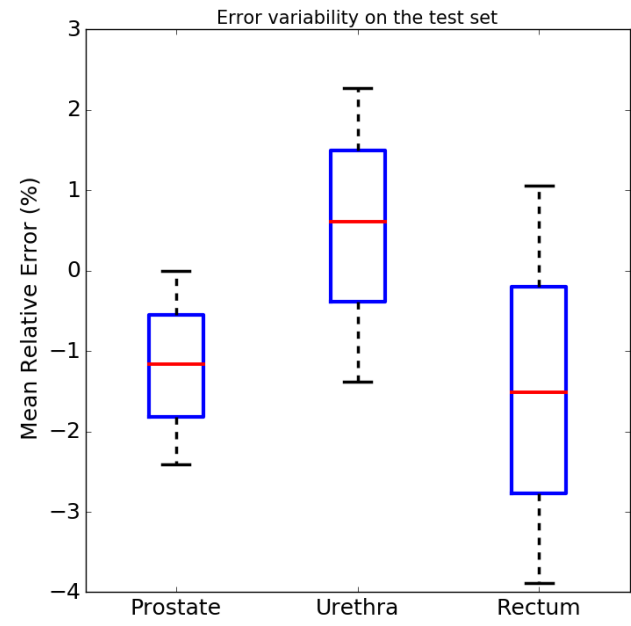

Fig. 6. Distribution of the mean relative error between DCNN predicted dose and MCS over the entire test set. The middle line represents the median, the central rectangle spans from the first quartile to the third quartile, and the vertical line extends from the first decile to the ninth decile

the figure 5 for the dose within the urethra, which appears to be clearly distinguishable in the MCS dose map but in the DCNN prediction the dose it is homogenized to the dose level of the neighboring region on the prostate. This is probably caused by the convolution operations that works on a filtering principle, and thus tend to smooth the changes of intensity. Despite the results on the error distribution and the qualitative observations, the dose prediction of the DCNN and the ground-truth are highly similar. Moreover, the final results on the inverse planning are not significantly affected by this prediction error, as could be stated in the DVH results.

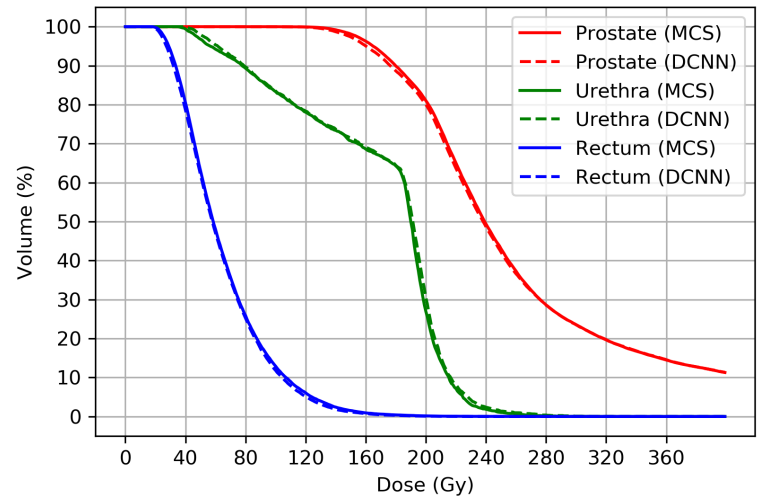

Fig. 7. DVH obtained after inverse planning on a test set patient by using our DCNN dose map calculation method.

Concerning the inverse planning quality results on the DVH parameters, our method tends to find optimal configurations with an under-estimated dose with respect to MCS on the prostate and rectum, whereas the DVH parameters on the urethra are slightly above the MCS values. However, these errors are below $1 \%, 2 \%$ and $3 \%$ for the prostate, urethra and rectum respectively. Yet, these errors are acceptable and still much lower than the TG-43 formalism which could reach the $12 \%$. Moreover, these errors do not affect the final outcome of the dose and all the DVH criteria are fulfilled on the recalculated MCS, which was the main purpose of our study.

Prediction time was highly reduced with respect to the MCS. For the same low level of uncertainty, we reached a dose map computation around 710 times faster than the GPU-based 


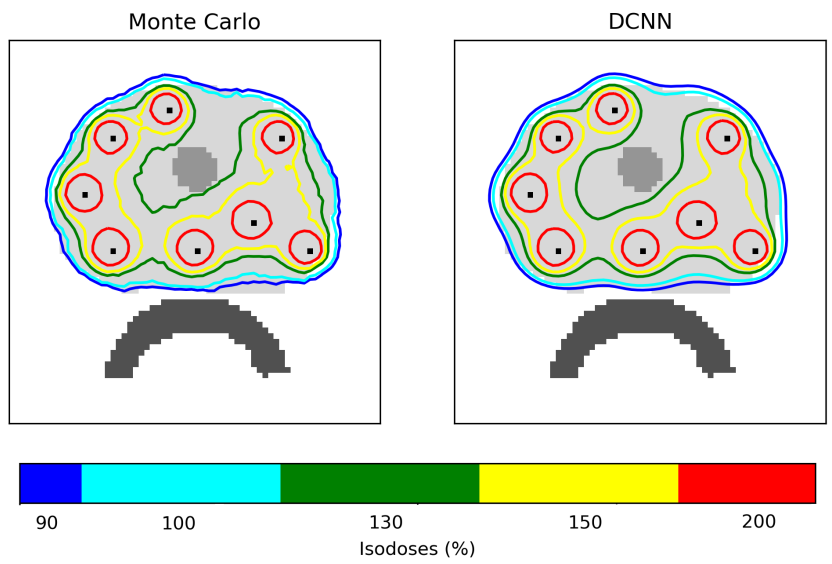

Fig. 8. Iso-dose distribution comparison on a mid-slice between the DCNN and the computed MCS after optimization. The values are represented as \% of the prescribed dose, which is equal to $160 \mathrm{~Gy}$.

\section{Monte Carlo simulation.}

One of the advantages of our DCNN approach compared to others of the state-of-the-art is that it is not dependent of the imaging system, due to the fact that it directly uses the annotated organ contours. However, a good segmentation must be guaranteed in order to avoid inaccuracies on the delivered dose, which is not the focus of our study. Finally, the most important innovation of our approach is that all the seeds' positions are encoded directly into the patient's image, which compared to previous approaches, prevents from estimating each seed effect separately and thus improving computation time and accuracy.

As a future work, we will study the applicability of our approach on focal brachytherapy. Additionally, we will study the feasibility of a DL technique for decrease the number of iterations needed by the FSA optimization.

\section{CONCLUSION}

We proposed a new DCNN-based method for performing DVH-based inverse planning dosimetry for LDR prostate brachytherapy. The DCNN model is capable to learn accurately from a MCS generated dose map database. Our approach is a potential solution for being adapted on the commercial inverse planning systems as an alternative calculation of the existing TG-43 formalism.

\section{ACKNOWLEDGMENT}

This work was funded by the French National Research Agency through the FOCUS project (ANR-16-CE19-0011), the OptimiX project (ANR-18-CE45-0011), and within the Investissements d'Avenir program (LabEx CAMI 2) under reference ANR-11-LABX-0004.

\section{REFERENCES}

[1] R. Nath, W. S. Bice, W. M. Butler, Z. Chen, A. S. Meigooni, V. Narayana, M. J. Rivard, and Y. Yu, "Executive Summary of Report of Task Group 137," Med. Phys., vol. 36, no. 11, pp. 5310-5322, 2009.
[2] E. Lessard and J. Pouliot, "Inverse planning anatomy-based dose optimization for HDR-brachytherapy of the prostate using fast simulated annealing algorithm and dedicated objective function," Med. Phys., vol. 28, no. 5, pp. 773-779, 2001.

[3] Y. Yu and M. C. Schell, "A genetic algorithm for the optimization of prostate implants," Medical Physics, vol. 23, no. 12, pp. 2085-2091, 1996.

[4] P. McGeachy, J. Madamesila, A. Beauchamp, and R. Khan, "An opensource genetic algorithm for determining optimal seed distributions for low-dose-rate prostate brachytherapy," Brachytherapy, vol. 14, no. 5, pp. 692-702, 2015.

[5] C. Guthier, K. P. Aschenbrenner, D. Buergy, M. Ehmann, F. Wenz, and J. W. Hesser, "A new optimization method using a compressed sensing inspired solver for real-time $\{\mathrm{LDR}\}$-brachytherapy treatment planning," Phys. Med. Biol., vol. 60, no. 6, pp. 2179-2194, 2015.

[6] M. J. Rivard, B. M. Coursey, L. A. DeWerd, W. F. Hanson, M. S. Huq, G. S. Ibbott, M. G. Mitch, R. Nath, and J. F. Williamson, "Update of AAPM Task Group No. 43 Report: A revised AAPM protocol for brachytherapy dose calculations," pp. 633-674, 2004.

[7] M. J. Rivard, W. M. Butler, L. A. DeWerd, M. S. Huq, G. S. Ibbott, A. S Meigooni, C. S. Melhus, M. G. Mitch, R. Nath, and J. F. Williamson, "Supplement to the 2004 update of the AAPM Task Group No. 43 Report," Med. Phys., vol. 34, no. 6, pp. 2187-2205, 2007.

[8] M. J. Rivard, J. L. Venselaar, and L. Beaulieu, "The evolution of brachytherapy treatment planning," Med. Phys., vol. 36, no. 6, pp. 2136 2153, 2009.

[9] J. F. Carrier, L. Beaulieu, F. Therriault-Proulx, and R. Roy, "Impact of interseed attenuation and tissue composition for permanent prostate implants," Med. Phys., vol. 33, no. 3, pp. 595-604, 2006.

[10] J. F. Carrier, M. D'Amours, F. Verhaegen, B. Reniers, A. G. Martin, É. Vigneault, and L. Beaulieu, "Postimplant Dosimetry Using a Monte Carlo Dose Calculation Engine: A New Clinical Standard," Int. J. Radiat. Oncol. Biol. Phys., vol. 68, no. 4, pp. 1190-1198, 2007.

[11] J. Sempau, S. J. Wilderman, and A. F. Bielajew, "DPM, a fast, accurate Monte Carlo code optimized for photon and electron radiotherapy treatment planning dose calculations," Phys. Med. Biol., vol. 45, no. 8, pp. 2263-2291, 2000 .

[12] S. Hissoiny, B. Ozell, H. Bouchard, and P. Després, "GPUMCD: A new GPU-oriented Monte Carlo dose calculation platform," Med. Phys., vol. 38, no. 2, pp. 754-764, 2011.

[13] J. Bert, H. Perez-Ponce, Z. E. Bitar, S. Jan, Y. Boursier, D. Vintache, A. Bonissent, C. Morel, D. Brasse, and D. Visvikis, "Geant4-based Monte Carlo simulations on GPU for medical applications," Phys. Med. Biol., vol. 58, no. 16, pp. 5593-5611, aug 2013.

[14] Y. Lemaréchal, J. Bert, C. Falconnet, P. Després, A. Valeri, U. Schick, O. Pradier, M. P. Garcia, N. Boussion, and D. Visvikis, "GGEMSBrachy: GPU GEant4-based Monte Carlo simulation for brachytherapy applications," Phys. Med. Biol., vol. 60, no. 13, pp. 4987-5006, 2015.

[15] K. A. Mountris, D. Visvikis, and J. Bert, "DVH-Based Inverse Planning Using Monte Carlo Dosimetry for LDR Prostate Brachytherapy Radiation Oncology," Int J Radiat. Oncol Biol Phys, vol. 103, no. 2, pp. 503-510, 2019. [Online]. Available: https://doi.org/10.1016/j.ijrobp. 2018.09.041

[16] X. Mao, J. Pineau, R. Keyes, and S. A. Enger, "RapidBrachyDL: Rapid Radiation Dose Calculations in Brachytherapy via Deep Learning," Int. J. Radiat. Oncol. Biol. Phys., 2020. [Online]. Available: https://doi.org/10.1016/j.ijrobp.2020.04.045

[17] M. Bethesda, "Icru report n. 46: Photon, electron, proton and neutron interaction data for body tissues," Tech. Rep, Tech. Rep., 1992.

[18] O. Chibani and J. F. Williamson, "MCPI@: A sub-minute Monte Carlo dose calculation engine for prostate implants," Med. Phys., vol. 32, no. 12, pp. 3688-3698, nov 2005.

[19] J. F. Williamson, "Monte Carlo evaluation of kerma at a point for photon transport problems," Medical Physics, vol. 14, no. 4, 1987. [Online]. Available: https://aapm.onlinelibrary.wiley.com/doi/abs/ $10.1118 / 1.596069$

[20] Ö. Çiçek, A. Abdulkadir, S. S. Lienkamp, T. Brox, and O. Ronneberger, "3D U-net: Learning dense volumetric segmentation from sparse annotation," in Lect. Notes Comput. Sci. (including Subser. Lect. Notes Artif. Intell. Lect. Notes Bioinformatics), vol. 9901 LNCS, 2016, pp. 424-432.

[21] D. P. Kingma and J. L. Ba, "Adam: A method for stochastic optimization," in 3rd Int. Conf. Learn. Represent. ICLR 2015 - Conf. Track Proc., vol. abs/1412.6, 2015.

[22] J. Pouliot, D. Tremblay, J. Roy, and S. Filice, "Optimization of permanent 125I prostate implants using fast simulated annealing," Int.J.Radiat.Oncol.Biol.Phys., vol. 36, no. 3, pp. 711-720, 1996. [Online]. Available: pm:8948357 